\title{
A Systems Approach to Drug Discovery in Alzheimer's Disease
}

\author{
Michael Shelanski • William Shin • Soline Aubry • \\ Peter Sims • Mariano J. Alvarez • Andrea Califano
}

Published online: 22 January 2015

(C) The American Society for Experimental NeuroTherapeutics, Inc. 2015 approaches in this second class has been successful, although trials on $\beta$ - or $\gamma$-secretase (BACE) inhibitors are ongoing.

The lack of overt success has been even more frustrating because, in transgenic (A $\beta$-overproducing) mouse models of $\mathrm{AD}, \mathrm{A} \beta$-lowering approaches, as well as treatments with small molecules and biologics, have been successful in blocking memory loss, restoring memory function, reversing dendritic spine alterations, and reversing inhibition of longterm potentiation [1-3]. Indeed, as animal models only partially recapitulate the human $\mathrm{AD}$ phenotype and because human brain tissue is available only postmortem, translation of preclinical findings to the clinics has been fraught with difficulties.

For instance, the characteristic intraneuronal neurofibrillary tangles and extensive neuronal loss observed in human $\mathrm{AD}$ are absent in mouse models of the disease. This dichotomy suggests that mouse models replicate only presymptomatic AD stages, while clinical manifestations appear only after neuronal loss becomes irreversible. This hypothesis is directly addressed by ongoing trials, where individuals with genetic mutations that predispose to early $\mathrm{AD}$ onset are being treated with $\mathrm{A} \beta$-targeted therapies to assess whether presymptomatic treatment may block an otherwise certain disease progression. Beyond $A \beta$-targeted therapies, novel approaches are being developed based on inhibition of tau phosphorylation and aggregation, and on blockade of synaptic loss, leveraging "candidate" target approaches derived from human and animal data. Unfortunately, it has so far been impossible to discern whether "candidate genes" represent causal determinants of the disease process or are the downstream result of them.

In this review, we will discuss the potential of adapting integrative systems biology approaches that have already proven extremely valuable in understanding multiple cancer related phenotypes to human neurodegenerative diseases. 


\section{Developing Human to Mouse to Human Approaches}

A key limitation of $\mathrm{AD}$ studies is that the mechanisms of disease initiation and progression, as well as their modulation using small molecule inhibitors, cannot be assessed in a human host. As a result, in vitro and in vivo models of $\mathrm{AD}$ have flourished. Yet, many of these models recapitulate only specific facets of the disease and, as a result, translation of findings from cell lines and transgenic mouse models to humans has been disappointing at best.

To address this impasse and to avoid findings that are idiosyncratic to a specific biological model but fail to generalize to the human context, we propose a cross-species approach that has been highly successful in the study of human malignancies [4]. Specifically, we propose assembling accurate, genome-wide regulatory models for both mouse models of $\mathrm{AD}$ and for their human counterpart to assess systematically the subset of the regulatory logic of $\mathrm{AD}$ neurons that is conserved or divergent between the two species. The rationale for this strategy is that investigation of therapeutic targets within conserved regulatory circuits will lead to successful translation from mouse models to human studies, while those embedded within a divergent cellular circuitry will prove to be idiosyncratic and will not be successfully translated.

Over the last 10 years there has been a veritable explosion of methodologies for the systematic reverse engineering of mammalian regulatory and signal transduction networks (henceforth interactomes for short) that are cell context specific and that have been thoroughly experimentally validated (see $[5,6]$ for a review). Murine interactomes including neuron-specific interactomes $[8,9]$, have been obtained by harvesting cells from mouse models representing diverse genetic backgrounds, tissues, and/or environmental stimuli, including small molecule perturbation in vivo [7]. For instance, to assemble an accurate murine interactome for cross-species analysis of prostate cancer (PC) progression, 13 distinct transgenic PC models were perturbed in vivo with 13 small molecule compounds and dimethyl sulfoxide to generate a set of 364 gene expression profiles from murine PC tissues and normal controls. These data were used to assemble a genomewide transcriptional regulatory model using the ARACNe algorithm $[10,11]$, where each transcription factor (TF) was associated with its $\mathrm{PC}$-specific transcriptional targets (i.e., the $T F$ regulon). The corresponding human $\mathrm{PC}$ interactome was produced by ARACNe analysis of a set of gene expression profiles from $\sim 200$ patient-derived PC samples, representing the full spectrum of disease progression. Comparison of the human and murine interactomes, using a novel algorithm, revealed that $70 \%$ of the regulatory programs in PC are highly conserved between these 2 species, including those of 2 synergistic master regulators (MRs) of progression to aggressive disease (forkhead box protein M1 and centromere protein F), inferred by the Master Regulator Inference algorithm (MARINa) [7, 12-15], and experimentally validated both in mouse and in human tissue. However, the analysis also showed that $30 \%$ of the programs are not conserved, including those representing a few PC-related genes that would thus be unlikely to produce patient-relevant results if studied or targeted in a murine context.

We propose that application of this approach to Alzheimer's disease will allow direct assessment of whether specific functional drivers and genetic determinants of human $\mathrm{AD}$ neurodegeneration, discovered by interrogating human neuronal interactomes with $\mathrm{AD}$ patient-derived signatures, are conserved in a mouse model of AD. This would allow their successful elucidation and targeting in a mouse context and the subsequent translation of relevant findings, including targeted therapeutics, back to a human context, implementing a bona fide human $\rightarrow$ mouse $\rightarrow$ human approach.

\section{Creating the Assembly Manual of the Alzheimer's Cell}

The mechanisms that regulate $\mathrm{AD}$ initiation and progression have been investigated largely without the benefit of an accurate and comprehensive map of the molecular interactions that underlie normal physiologic neuronal activity and, ultimately, its pathological dysregulation prior to neuronal demise in $\mathrm{AD}$. Existing regulatory and pathway models are inaccurate, sparse, and, most importantly, not representative of neuronand $\mathrm{AD}$-specific biology. In particular, canonical pathways, which are generally represented as universal rather than cellspecific models, are largely inadequate for the task of modeling the mechanism of disease initiation and progress. For instance, they fail to represent the cross-species diversity and intraspecies redundancy of signaling pathways, which are critical reasons why therapies do not translate effectively from the bench to the clinic or from model organisms to human biology.

Over the last few years, we have pioneered computational and experimental methods for the accurate dissection of tissue- and cell-specific molecular interaction networks, including those controlling transcriptional(protein-DNA)[10, $11,16,17]$, post-transcriptional(RNA-RNA and proteinRNA) [18-21], signal transduction and other posttranslational processes protein-protein interactions (PPI and metabolic) [22-26], and drug interactions [27-30]. These methods and datasets allow for the reconstruction of the regulatory and signaling logic of specific cell types by combining specific knowledge about regulatory mechanisms (e.g. transcription factors regulating expression, etc.) and information theoretic [11, 25], Bayesian [31, 32], and other machine learning methodologies [33] that can be used to infer causality and remove indirect interactions. Following reverse engineering analysis with these algorithms, each TF signaling protein 
and microRNA represented in an interactome is causally associated with a regulon containing dozens to hundreds of highly accurate and cell-context-specific targets and substrates. For instance, predictions by the ARACNe, MINDy, PrePPI algorithms have been typically validated at a very high rate $(\sim 70 \%)$ by chromatin immunoprecipitation, gene expression profiling following silencing, coimmunoprecipitation and other relevant assays [12, 19, 24, 25, 34, 35]. Other systems approaches have also been applied successfully in AD [36-39], and our focus here is to maintain clarity rather than review the entire literature. Each approach has a series of advantages and disadvantages. The approach outlined here is unbiased, not dependent on a large number of transcriptomes to detect changes between control and diseased tissues, and predicts alterations in transcription factors and transcriptional regulators that themselves might not be altered in the transcriptome.

Critically, these regulons provide the computational equivalent of a highly accurate gene reporter assay to measure the activity of the corresponding protein or other regulatory gene product. The MARINa algorithm was developed to infer the regulators that are causally responsible for implementing a phenotype-specific gene expression signature initially from multiple gene expression profiles $[12,14]$, and, more recently, even from a single gene expression profile [7, 40]. This is accomplished by measuring the enrichment of over- and underexpressed genes in positively regulated and repressed targets in the regulon of every possible regulator protein represented in the interactome, thus providing an accurate and extremely robust predictor of the differential activity of a regulator. This has allowed the discovery of key functional drivers of tumorigenesis and drug sensitivity, including single regulators $[13,15,41-43]$, synergistic regulator pairs $[4,12$, 14], and additive mechanisms that could not have been elucidated using traditional methods. Indeed, virtually none of the regulators that were experimentally validated were significantly differentially expressed at the RNA level and yet they were confirmed as individual or synergistic phenotypic drivers following identification by regulatory network analysis, thus elucidating novel mechanisms of disease initiation/ progression [4, 12, 13, 41-46], chemosensitivity [15, 28], and normal physiologic regulation [14]. Lately, we have successfully extended these methodologies to the study of neurological, neurodevelopmental, and stem cell phenotypes [35, $42,47]$, and, more recently, to neurodegenerative phenotypes, resulting in a series of manuscripts, currently in review, where we report on the elucidation and experimental validation of novel genes mediating neurotoxicity in ALS [48], as well as biomarkers of $\mathrm{AD}$ progression [9].

More recently, we have shown that MARINa-inferred MRs represent tight regulatory bottlenecks that integrate a large spectrum of germline variants and somatic alterations in upstream pathways [40]. These bottlenecks allow genetic variants and alterations patterns that are substantially distinct in different patients to converge on activating or inactivating key genetic programs that represent the hallmarks of the disease. Indeed, by traversing pathways upstream of MARINa-inferred MRs, using the MINDy algorithm, we identified the vast majority of established germline variants and genetic alterations that causally associated with glioma and breast cancer tumorigenesis, as well as $\mathrm{AD}$ predisposition. These alterations were not statistically significant by genome wide association studies. In addition, novel alterations predicted by the methodology for the mesenchymal subtype of high-grade glioma were experimentally validated as the most frequent causal determinants of the disease subtype [40]. Critically, this analysis could be performed on an individual sample basis, thus completely avoiding mechanism heterogeneity as a confounding factor that would negatively affect discovery when averaging on patients representing distinct mechanisms (e.g., all AD-affected individuals). In $\mathrm{AD}$, the method identified 14 predisposition variants, including known ones, such as $A P O E$ and TYROBP, among others. Surprisingly, 3 of 14 predisposition alleles identified by the algorithm were in the integrin pathway, including $I T G B 2$, ITGBL1, and ITGAM, which has been functionally linked to aberrant tau and $A \beta$ activity but never previously linked to $\mathrm{AD}$ in terms of risk predisposition alleles.

Finally, these methodologies have proven extremely valuable in elucidating context specific mechanisms of action of small molecules, as well as their activity in cells both individually and in combination $[28,49]$, thus paving the road to the mechanism-based identification of both single agent and combination therapy. While some are still in review, the results included in these manuscripts suggest that the regulatory network analysis strategies developed for cancer are eminently applicable to neurodegenerative diseases.

\section{Development of a Quantitative Systems Pharmacology Approach for the Identification of Molecular Targets and Associated Small Molecule Modulators}

There are several criticisms of traditional high-throughput screening (HTS) assays to identify small molecules that may be useful in AD. First, HTS approaches are limited to use in in vitro models of a disease, often using a reporter that is only indirectly linked to the human relevant phenotype. Second, results obtained using in vitro disease models rarely translate to an in vivo context. The latter is due to a variety of reasons: from the 3-dimensional versus 2-dimensional nature of the culture, to the presence of critical signals from other cellular niches in the neural microenvironment, to the presence of local paracrine and distal endocrine signals as well as other factors.

To address these limitations, a radically different approach has been proposed. In this approach, one first identifies candidate MRs of $\mathrm{AD}$ pathogenesis from $\mathrm{AD}$ patient gene signatures. This can be accomplished by interrogating a human 
neuron-specific interactome, using the MARINa algorithm as discussed in the previous section, with signatures of disease progression using gene expression profiles from normal and demented AD patients. We have tested this approach for its feasibility by generating a human neuronal interactome using transcriptional data generated from laser-dissected control, nondemented and $\mathrm{AD}$ human neurons from multiple regions of postmortem brains (GSE5281 and GSE9770) [50-52]. This interactome was then queried using gene expression profiles from AD and control tissues. Alterations in YY1, p300, and ZMYM3 were predicted by this approach and then validated by immunohistochemistry in $\mathrm{AD}$ brains and in $\mathrm{A} \beta$-treated rat primary hippocampal neurons. These represent novel biomarkers and potential therapeutic targets for the disease. A number of other predicted MRs are currently under study and a paper is currently under review [9].

Candidate MRs will need further validation-first in vitro, by RNA interference or clustered regularly interspaced short palindromic repeats-based silencing in neurons, and then in vivo, following cross-species analysis to identify an appropriate model — to assess whether they truly control the predicted genetic programs that are dysregulated in AD. Validated MRs will then be evaluated as candidate functional targets of both single compounds and compound combinations, using a nonredundant library of US Food and Drug Administration-approved drugs and probe/toolkit compounds. A powerful new screening tool is a novel technology for Pooled Library Amplification for Transcriptome Expression (PLATE-Seq), recently developed in the Sims lab at Columbia University, to perform multiplexed, barcoded RNA-Seq of 96 samples as a single library, thus achieving a cost of \$20-30 per sample at a depth of $2 \mathrm{M}$ reads. In PLATE-Seq, well-specific barcodes are introduced during reverse transcription. Thus, complementary DNA libraries from individual wells can be pooled immediately after reverse transcription, such that all subsequent bead- and column-based isolations steps, second strand synthesis, and polymerase chain reaction can take place on a single, pooled sample. This approach dramatically simplifies generation of large-scale gene expression signatures following perturbation with relatively large libraries of US Food and Drug Administration-approved and experimental compounds. In cellular systems, for which an ARACNe-based interactome is available, PLATE-Seq libraries can be used to assess compound effectors and targets with no fidelity loss compared with $30 \mathrm{M}$ read RNA-Seq owing to the integration of expression values over each protein's regulon. Taken together with the low coverage requirements of our algorithms, the reduction in reagent cost and hands-on time afforded by PLATE-Seq allows generation of genome-wide expression signature on an unprecedented scale.

Such an approach can be used to screen cells following perturbation with compound libraries, thus helping elucidate the compound effectors and substrates using the same MARI $\mathrm{Na}$ algorithm and the same regulatory models used for $\mathrm{AD}$
MR inference. Importantly, as this analysis can be aimed at identifying modulators of specific protein activities, rather than AD-relevant endpoint phenotypes, it is well suited to in vitro analysis as compound substrates and targets are generally well conserved in vitro and in vivo. This approach can thus be used to prioritize all library compounds that are likely to reverse the specific pattern of aberrant MR activation and inactivation observed in demented AD patients, as well as to determine their full activity and mechanism of action, thus identifying potential toxicity issues. A novel algorithm (SynGen) was developed to assess the potential synergy of 2 compounds in implementing a specific target phenotype using such single-compound RNA-Seq signatures. The analysis was tested against a public database as part of the dialogue for reverse engineering assessments and methods (DREAM) challenge on drug synergy, and was effective in predicting $60 \%$ of the experimentally assessed synergistic compound combinations at an $8 \%$ false discovery rate [28].

We propose that such a hybrid experimental/computational methodology will allow identification of optimal compounds and compound combinations that will help revert the MR signatures detected in $\mathrm{AD}$ patients toward a more normal physiologic state. Top single agents and compound combinations emerging from the analysis would be tested in increasingly specific models and finally in the murine models of $\mathrm{AD}$ assessed to be most human-relevant, based on cross-species network analysis. Specifically, these analyses will test whether the specific mechanisms targeted by compounds or compound combinations are a) conserved in the mouse and b) equivalently targeted by the small molecule in the mouse.

\section{Approaches to Validation}

Validation of MRs can be performed by established techniques, using a) phenotypic assays in vitro and in vivo, and b) molecular assays based on PlateSeq or Fluidigm multiplexed quantitative polymerase chain reaction, to determine whether RNA interference or clustered regularly interspaced short palindromic repeats-based modulation of individual MRs and predicted synergistic MR pairs will induce the predicted regulatory outcome and phenotypic end points. Such efforts will produce a set of validated MRs that will be used to screen for specific small molecule compound activity. Compounds will thus be prioritized based on their ability to abrogate the aberrant activity of validate MRs based on analysis of their key effectors following perturbation of neuronal cells, as assessed by analysis of PlateSeq-based RNA-Seq profiles of perturbed cells.

Small molecule modulators (either as single compound or synergistic compound pairs) can be prioritized through these quantitative model-based approaches and then be experimentally validated. This is accomplished by the establishment of robust and reproducible correlative profiles between 
compound-induced changes in $\mathrm{AD}$-specific expression signatures (using the validated MRs) versus functional changes in our available phenotypic assays using in vitro mouse and human model as well as in vivo mouse models. For in vitro models, models include dissociated mouse primary neurons or embryonic stem cell-derived pyramidal neurons derived from the Tg2576 mouse model of AD. HTS assays exist for cell-associated production of SAPP $\beta$ [BACE1 cleavage product of amyloid precursor protein (APP)] in neuronal cells, $A \beta$ induced redistribution of intracellular tau in embryonic stem cell-derived neurons, and apolipoprotein E secretion from human astrocytes. Additional assays are also available for follow-up validation, including $A \beta$-induced synaptic dysfunction using multiple measures of electrophysiology, biochemistry, and morphology, in primary neurons as well as ex vivo brain slice models. AD-relevant phenotypic assays in human induced pluripotent stem cells (hiPS)-derived neurons for $A \beta$-triggered neuronal death and synaptic deficits are also being developed. Validation end points will be reversal of MR activity in these cellular assays, as assessed by the MARINa analysis of RNA-Seq profiles following compound perturbation, as well as surrogate phenotypic readouts, such as dendritic spine density, caspase activation or tau phosphorylation. Small molecule modulators that are validated using these in vitro models can then be tested in well-characterized animal models to determine AD-relevant preclinical efficacy. Potential model systems include transgenic and nontransgenic mouse models of $\mathrm{AD}$, including the APP/PS1 mouse model of amyloid elevation [53, 54], the hTau/Tau knockout transgenic mouse line in which the mouse tau gene is replaced by the nonmutated human tau gene $[55,56]$, and mice exposed to $A \beta$ and tau oligomers through cannulas implanted onto dorsal hippocampi as nontransgenic models of $\mathrm{AD}$ [57]. These animals can be treated with a given compound/s as indicated by the experiments on in vitro models, and then undergo long-term potentiation assessment of synaptic function and behavioral testing with a battery of cognitive tasks, including fear conditioning, radial arm water maze, and the Morris water maze [57]. The successful completion of this step will establish the compound-target and target-disease networks that are functioning through the diverse mechanisms, including the one target-one compound paradigm, as well as polypharmacology.

These approaches have the potential to rapidly improve the route to AD drug discovery. The ability to screen on the reversal of effects on MRs before going into time-consuming animal models makes the approach both effective and economically sensible.

Required Author Forms Disclosure forms provided by the authors are available with the online version of this article.

\section{References}

1. Gong B, Cao Z, Zheng P, et al. Ubiquitin hydrolase Uch-L1 rescues beta-amyloid-induced decreases in synaptic function and contextual memory. Cell 2006;126:775-788.

2. Smith DS, Pozueta J, Gong B, et al. Reversal of Long-Term Dendritic Spine Alterations in Alzheimer Disease Models. Proc Nat Acad Sci (US) 2009;106:16877-16882

3. Shrestha BR, Vitolo OV, Joshi P, Lordkipanidze T, Shelanski M, Dunaevsky A. Amyloid beta peptide adversely affects spine number and motility in hippocampal neurons. Mol Cell Neurosci 2006;33: 274-282.

4. Aytes A, Mitrofanova A, Lefebvre C, et al. Cross-species analysis of genome-wide regulatory networks identifies a synergistic interaction between FOXM1 and CENPF that drives prostate cancer malignancy. Cancer Cell 2014;25:638-651.

5. Califano A, Butte AJ, Friend S, Ideker T, Schadt E. Leveraging models of cell regulation and GWAS data in integrative networkbased association studies. Nat Genet 2012;44:841-847.

6. Lefebvre C, Rieckhof G, Califano A. Reverse-engineering human regulatory networks. Wiley Interdiscip Rev Syst Biol Med 2012;4: 311-325.

7. Aytes A, Mitrofanova A, Lefebvre C, et al. Cross-species regulatory network analysis identifies a synergistic interaction between FOXM1 and CENPF that drives prostate cancer malignancy. Cancer Cell 2014;25:638-651.

8. Repunte-Canonigo V, Shin W, Vendruscolo LF, et al. Reverse engineering of transcriptional networks identifies critical neuroadaptations of the glucocorticoid receptor that mediate excessive alcohol drinking during protracted withdrawal from alcohol dependence. Genome Biol 2014 (in press).

9. Aubry S, Shin W, Lefort, R, et al. Reverse engineering of Alzheimer's disease neuronal transcriptional networks - Validated targets point to the loss of histone acetylation homeostasis in the disease progression. Soc Neurosci 2015:228

10. Basso K, Margolin AA, Stolovitzky G, Klein U, Dalla-Favera R, Califano A. Reverse engineering of regulatory networks in human B cells. Nat Genet 2005;37:382-390.

11. Margolin AA, Nemenman I, Basso K, et al. ARACNE: an algorithm for the reconstruction of gene regulatory networks in a mammalian cellular context. BMC Bioinform 2006;7(Suppl. 1):S7.

12. Carro MS, Lim WK, Alvarez MJ, et al. The transcriptional network for mesenchymal transformation of brain tumours. Nature 2010;463: 318-325.

13. Chudnovsky Y, Kim D, Zheng S, et al. ZFHX4 interacts with the NuRD core member CHD4 and regulates the glioblastoma tumorinitiating cell state. Cell Rep 2014;6:313-324.

14. Lefebvre C, Rajbhandari P, Alvarez MJ, et al. A human B-cell interactome identifies MYB and FOXM1 as master regulators of proliferation in germinal centers. Mol Syst Biol 2010;6:377.

15. Piovan E, Yu J, Tosello V, et al. Direct reversal of glucocorticoid resistance by AKT inhibition in acute lymphoblastic leukemia. Cancer cell. 2013;24:766-776.

16. Margolin AA, Wang K, Lim WK, Kustagi M, Nemenman I, Califano A. Reverse engineering cellular networks. Nat Protocol 2006;1:662671.

17. Lee TI, Rinaldi NJ, Robert F, et al. Transcriptional regulatory networks in Saccharomyces cerevisiae. Science 2002;298:799-804.

18. Chiu HS, Llobet-Navas D, Yang X, et al. Cupid: simultaneous reconstruction of microRNA-target and ceRNA networks. Genome Res 2014 Nov 5 [Epub ahead of print].

19. Sumazin P, Yang X, Chiu HS, et al. An extensive microRNAmediated network of RNA-RNA interactions regulates established oncogenic pathways in glioblastoma. Cell 2011;147:370-381. 
20. Janga SC, Vallabhaneni S. MicroRNAs as post-transcriptional machines and their interplay with cellular networks. Adv Exp Med Biol 2011;722:59-74.

21. Janga SC, Mittal N. Construction, structure and dynamics of posttranscriptional regulatory network directed by RNA-binding proteins. Adv Exp Med Biol 2011;722:103-117.

22. Jansen R, Yu H, Greenbaum D, et al. A Bayesian networks approach for predicting protein-protein interactions from genomic data. Science 2003;302:449-453.

23. Palsson B. Metabolic systems biology. FEBS Lett 2009;583:3900 3904.

24. Zhang QC, Petrey D, Deng L, et al. Structure-based prediction of protein-protein interactions on a genome-wide scale. Nature 2012;490:556-560.

25. Wang K, Saito M, Bisikirska BC, et al. Genome-wide identification of post-translational modulators of transcription factor activity in human B cells. Nat Biotechnol 2009;27:829-839.

26. Rual JF, Venkatesan K, Hao T, et al. Towards a proteome-scale map of the human protein-protein interaction network. Nature 2005;437: 1173-1178.

27. di Bernardo D, Thompson MJ, Gardner TS, et al. Chemogenomic profiling on a genome-wide scale using reverse-engineered gene networks. Nat Biotechnol 2005;23:377-383.

28. Bansal M, Yang J, Karan C, et al. Predicting compound synergy from first principles, a crowdsourcing approach. Nature Biotechnol 2014;32:1213-1222..

29. Basu A, Bodycombe NE, Cheah JH, et al. An interactive resource to identify cancer genetic and lineage dependencies targeted by small molecules. Cell 2013;154:1151-1161.

30. Costello JC, Heiser LM, Georgii E, et al. A community effort to assess and improve drug sensitivity prediction algorithms. Nat Biotechnol 2014;32:1202-1212.

31. Sachs K, Perez O, Pe'er D, Lauffenburger DA, Nolan GP. Causal protein-signaling networks derived from multiparameter single-cell data. Science 2005;308:523-529.

32. Friedman N. Inferring cellular networks using probabilistic graphical models. Science 2004;303:799-805.

33. Kwong LN, Costello JC, Liu H, et al. Oncogenic NRAS signaling differentially regulates survival and proliferation in melanoma. Nat Med 2012;18:1503-1510.

34. Basso K, Saito M, Sumazin P, et al. Integrated biochemical and computational approach identifies BCL6 direct target genes controlling multiple pathways in normal germinal center B cells. Blood 2010;115:975-984.

35. Kushwaha R, Jagadish N, Kustagi M, et al. Interrogation of a contextspecific transcription factor network identifies novel regulators of pluripotency. Stem Cells 2014 Oct 21 [Epub ahead of print].

36. Zhang B, Gaiteri C, Bodea LG, et al. Integrated systems approach identifies genetic nodes and networks in late-onset Alzheimer's disease. Cell 2013;153:707-720.

37. Miller JA, Horvath S, Geschwind DH. Divergence of human and mouse brain transcriptome highlights Alzheimer disease pathways. Proc Natl Acad Sci U S A 2010;107:12698-12703.

38. Rhinn H, Fujita R, Qiang L, Cheng R, Lee JH, Abeliovich A. Integrative genomics identifies APOE epsilon4 effectors in Alzheimer's disease. Nature 2013;500:45-50.

39. Rosen EY, Wexler EM, Versano R, et al. Functional genomic analyses identify pathways dysregulated by progranulin deficiency, implicating Wnt signaling. Neuron 2011;71:1030-1042.
40. Chen JC, Alvarez MJ, Talos F, et al. Identification of causal genetic drivers of human disease through systems-level analysis of regulatory networks. Cell 2014;159:402-414.

41. Della Gatta G, Palomero T, Perez-Garcia A, et al. Reverse engineering of TLX oncogenic transcriptional networks identifies RUNX1 as tumor suppressor in T-ALL. Nat Med 2012;18:436-440.

42. Zhao X, D'Arca D, Lim WK, et al. The N Myc DLL3 cascade is suppressed by the ubiquitin ligase Huwel to inhibit proliferation and promote neurogenesis in the developing brain. Developmental Cell 2009; 17(2):210-221.

43. Compagno M, Lim WK, Grunn A, et al. Mutations of multiple genes cause deregulation of NF-kappaB in diffuse large B-cell lymphoma. Nature 2009;459:717-721.

44. Bisikirska BC, Adam SJ, Alvarez MJ, et al. STK38 is a critical upstream regulator of MYC's oncogenic activity in human B-cell lymphoma. Oncogene 2013;32:5283-5291.

45. De Keersmaecker K, Real PJ, Gatta GD, et al. The TLX1 oncogene drives aneuploidy in T cell transformation. Nat Med 2010;16:13211327.

46. Palomero T, Lim WK, Odom DT, et al. NOTCH1 directly regulates c-MYC and activates a feed-forward-loop transcriptional network promoting leukemic cell growth. Proc Natl Acad Sci U S A 2006;103:18261-18266.

47. Chen J, Repunte-Canonigo V, Kawamura T, et al. Hypothalamic proteoglycan syndecan-3 is a novel cocaine addiction resilience factor. Nat Commun 2013;4:1955.

48. Ikiz B, Alvarez MJ, Re DB, et al. Dissecting the regulatory machinery of neurodegeneration in an in vitro model of amyotrophic lateral sclerosis. in review. 2014.

49. Mani KM, Lefebvre C, Wang K, Lim WK, Basso K, Dalla-Favera R, Califano A. A systems biology approach to prediction of oncogenes and molecular perturbation targets in B-cell lymphomas. Mol Syst Biol 2008;4:169.

50. Liang WS, Dunckley T, Beach TG, et al. Altered neuronal gene expression in brain regions differentially affected by Alzheimer's disease: a reference data set. Physiol Genom 2008;33:240-256.

51. Liang WS, Dunckley T, Beach TG, et al. Neuronal gene expression in non-demented individuals with intermediate Alzheimer's Disease neuropathology. Neurobiol Aging 2010;31:549-566.

52. Liang WS, Dunckley T, Beach TG, et al. Gene expression profiles in anatomically and functionally distinct regions of the normal aged human brain. Physiol Genom 2007;28:311-322.

53. Holcomb L, Gordon MN, McGowan E, et al. Accelerated Alzheimertype phenotype in transgenic mice carrying both mutant amyloid precursor protein and presenilin 1 transgenes. Nat Med 1998;4:97100.

54. Trinchese F, Liu S, Battaglia F, Walter S, Mathews PM, Arancio O. Progressive age-related development of Alzheimer-like pathology in APP/PS1 mice. Ann Neurol 2004;55:801-814.

55. Andorfer C, Kress Y, Espinoza M, et al. Hyperphosphorylation and aggregation of tau in mice expressing normal human tau isoforms. $\mathrm{J}$ Neurochem 2003;86:582-590.

56. Polydoro M, Acker CM, Duff K, Castillo PE, Davies P. Agedependent impairment of cognitive and synaptic function in the htau mouse model of tau pathology. J Neurosci 2009;29: 10741-10749.

57. Puzzo D, Lee L, Palmeri A, Calabrese G, Arancio O. Behavioral assays with mouse models of Alzheimer's disease: practical considerations and guidelines. Biochem Pharmacol 2014;88:450-467. 